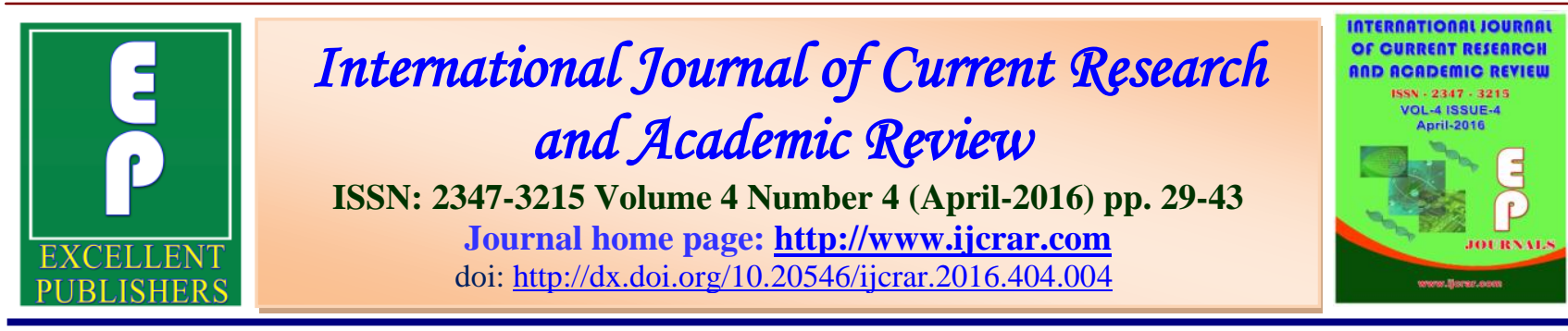

\title{
A Review on Amelioration of Mushroom Tyrosinase for Phenol Degradation to Combat Environment Pollution
}

\section{Anjana Sharma* and Juhi Sharma}

Bacteriology Laboratory, Department of P. G. Studies and Research in Biological Science, Rani Durgavati University, Pachpedi, Jabalpur 482001, Madhya Pradesh, India

*Corresponding author

\begin{tabular}{|c|c|}
\hline KEYWORDS & A B S T R A C T \\
\hline $\begin{array}{l}\text { Mushroom, } \\
\text { Purification, } \\
\text { Tyrosinase, } \\
\text { Phenol } \\
\text { Degradation. }\end{array}$ & $\begin{array}{l}\text { Mushroom tyrosinase would appear to be natural contenders for the formation } \\
\text { of commercial processes, based on the statistic that many of the laboratory } \\
\text { lessons have involved enzymes from this group of organisms. The current } \\
\text { review momentarily synopses the existing state of the art in relation to } \\
\text { production and purification of mushroom tyrosinase. Tyrosinase has the } \\
\text { benefit that it can react with molecular oxygen without the need for } \\
\text { externally-supplied co-substrates, which leads to subordinate costs. Further, } \\
\text { when tyrosinase oxidizes phenols and other aromatic compounds in } \\
\text { wastewaters, typically the oxidized product will polymerize to insoluble } \\
\text { compounds that can be detached by filtration or precipitation. Due to these } \\
\text { features, tyrosinase has been considered for phenol removal from } \\
\text { wastewaters. Presence of mushroom tyrosinase, innumerable methods for its } \\
\text { purification and Enzymatic method for amputation of phenols from industrial } \\
\text { waste water is reviewed. }\end{array}$ \\
\hline
\end{tabular}

\section{Introduction}

Phenolic compound emancipation grounds environment pollution. Mushroom Tyrosinase is decidedlydiscerning and efficient for phenol treatment. Purified tyrosinase has foremost solicitation in dephenolization

\section{Phenolic Pollution in Environment}

Phenol is contemporary in the wastewater from a number of industries, including coal conversion, petroleum refinery, mining, and dressing operations, and facilities producing resins, plastics, dyes and other organic chemicals, textiles, timber, and pulp and paper. Phenol ranks among the top 25 chemicals with the largest waste transfer and ranks among the 25 chemicals with the largest national pollutant release inventory and toxic release inventory (TRI) transfers. It is also among the top 10 TRI chemicals released to sewage or Publicly Owned Treatment Works. Virtually all phenols are toxic; furthermore, phenol and many of its 
derivatives are hazardous pollutant phenol concentrations greater than $50 \mathrm{ppb}$ are toxic to some forms of aquatic life and ingestion of $1 \mathrm{~g}$ of phenol can be fatal in humans. Hence, due to their abundance and toxicity, removal of phenols from industrial aqueous effluents is an important practical problem (Toxic release inventory (TRI) transfers report 2015).Kulkarni and Kaware (2013) concluded that phenol is a major pollutant in the wastewater because of its presence in the effluent of major processing and refining plants. It has severe effect on human being, both short term and long term. Suitable method for phenol removal can be selected based on availability of the material, extent of separation required and properties of phenolic effluent. As contamination in groundwater and its subsequent health effects has become a worldwide concern over the past two to three decades. Untreated, highly toxic as in soil and effluent has been disposed of in rivers and has ended up in groundwater, leading to toxicity to humans and other biota (Paul et al., 2015).

Koshy et al. in (2012) through their study resulted that phenol is an aromatic hydrocarbon which exists as a colorless or white solid in its pure state. Over the past several decades, there is growing concern about wide spread contamination of surface and ground water by phenol, due to rapid development of chemical and petrochemical industries. Phenol affects aquatic life even at relatively low concentration $(5-25 \mathrm{mg} / \mathrm{L})$. Treatment for removal of phenol includes chemical as well as biological processes. Studies show that ligninases such as Lignin Peroxidase and Laccase, produced by Pleurotus sp., can degrade phenol. Spent substrate of Pleurotus mushrooms consists of ligninases. After harvest, spent substrate was utilized for phenol degradation. From these studies it appears that, spent substrate of $P$. ostreatus can be used effectively to remove phenol from industrial effluents.

\section{Dephenolization Techniques}

Conventional methods for dephenolization of industrial wastewaters include solvent extraction, deep-well injection, microbial degradation, adsorption on activated carbon and chemical oxidation. Solvent extraction methods are expensive and deep-well injection may lead to contamination of ground water. Adsorption and oxidation treatments become exceedingly expensive when low effluent concentrations must be achieved. Microbiological treatment has shown great promise, but for small volume wastes generated discontinuously, microbiological treatment has been plagued by instabilities caused by toxicity of these compounds to the microbial population. Therefore, alternative technologies have to be explored. Enzymatic treatment of phenolics was of particular advantage of enzymatic treatment is the high specificity of enzymes for their substrates, unlike microbiological processes, which tend to be non-selective (Flurkey and Siu, 2006). However, enzymes can be readily inactivated, dramatically increasing the cost of an enzyme-based process for phenol removal. Tyrosinase, one enzyme suitable for treatment of phenolic wastes, catalyzes two consecutive reactions via separate active sites: Hydroxylation of monophenols with molecular oxygen in the presence of a chemical reductor (AH2) to form orthodiphenols, and Dehydrogenation of orthodiphenols with molecular oxygen to form ortho-quinones Quinones are usually formed rapidly, and undergo non-enzymatic conversion to form more stable intermediates. These intermediates subsequently undergo slow oligomerization reactions that ultimately yield high molecular weight, insoluble polyphenolics. At low used soluble 
tyrosinase to remove phenol from an aqueous synthetic waste solution; up to $99 \%$ conversion of the phenol was obtained, although, at higher concentrations $(1.0 \mathrm{~g} / \mathrm{L})$, conversion was limited by inactivation of tyrosinase, likely by quinines formed during the reaction. Hence, they suggested the use of chitosan to adsorb quinones generated during phenol degradation. Given that immobilization has the potential to increase enzyme stability, various researchers have examined the effectiveness of immobilized tyrosinase for phenol degradation. Phenols dissolved in chloroform could be degraded using tyrosinase immobilized on glass beads. Although enzyme stability was improved by immobilization or by incorporation in organic solvents (half-life of several hours versus several minutes for tyrosinase in aqueous solution), further improvements are required (Konishi et al., 2007).

Aromatic compounds are present in the wastewaters of a large number of industries including coal conversion, petroleum refining, resins and plastics, wood preservation, dyes, chemicals and textiles. The use of horseradish peroxidase and hydrogen peroxide to accomplish the removal of phenols and aromatic amines from these wastewaters has attracted much attention since the initial work. Treatment of aromatic substrates was accomplished through the oxidation of substrates by hydrogen peroxide under the influence of the biocatalyst. The free radical reaction products spontaneously react to form polymeric products of reduced solubility, which can be separated from solution by coagulation and subsequent sedimentation (Schroder et al., 2006). While peroxidase enzymes have the potential to treat a large variety of phenolic compounds over wide ranges of $\mathrm{pH}$ and temperature, the major concern regarding this enzymatic treatment is the prohibitive cost of the enzyme and hydrogen peroxide. One possible alternative to peroxide, tyrosinase (EC 1.14.18.1) which is widely distributed in bacteria, fruits, vegetables and sea food products. This type of enzyme, also known as polyphenol oxidase, catalyzes the oxidation of phenols but uses molecular oxygen as an oxidant. Ability to protect tyrosinase from inactivation reported that chitosan accelerated the rate of oxidation of several phenols. It was concluded that chitosan interacts with reaction products which may otherwise have inhibited or inactivated tyrosinase. While they demonstrated that the rate of reaction was significantly improved in the presence of chitosan, it was not quantify how much this resulted in a reduction in the quantity of tyrosinase required to achieve a given degree of transformation of phenolic substrates (Ikehata and Nicell, 2000).

\section{Mushroom Tyrosinase for Phenol Degradation}

Tyrosinase

(monophenols, dihydroxyphenylalanine, oxygen oxidoreductase, EC 1.14.18. I) is found throughout plant and fungal species. The enzyme from mushrooms has acknowledged substantial attention within the last 10 years. Most of this thoughtfulness and ensuing soundings have relied on commercial sources for their studies. Demonstrative topics in these studies have encompassed examination of inhibitor effects using methimazole, mimosine tropolone, ascorbic acid and its derivatives, cysteine and 3amino t-tyrosine (Konishi et al., 2007). Many studies have used commercial tyrosinase to explore new substrates such as phosphonic analogue of tyrosine and dopa, 1-naphthol, oxidation of 3, 4dihydroxymandelic acid, oxidation of dopaquinone addition products, and novel 
substrates. Some investigators have used the commercial enzyme to examine the effects of various compounds on the enzyme or kinetic characteristics of tyrosinase.

Three unusual enzyme activities have also been recently attributed to the mushroom enzyme. These include the oxidative dimerization of 1, 2-dehydro-nacetyldopamine, oxidative decarboxylation of 3,4-dihydroxymandelate and ferroxidase activity. Many reports used commercial preparations although Boyer et al. used a partially purified tyrosinase from commercial sources. The commercial enzyme has also been used for studies involving enzymatic reactions in organic media, affinity chromatography, and development of new assays for the enzyme and control of browning in processed mushrooms. With few exceptions, most of the above investigations have not reported or examined the isoenzyme content of tyrosinase in these preparations, nor have they examined the purity of the enzyme preparations. These factors are important because different isoenzyme forms could display specific inhibitor and kinetic characteristics. In addition, impure preparations could contain other enzymes capable of interfering with or catalyzing ancillary reactions utilizing potential substrates or inhibitors of tyrosinase (Konishi et al., 2007).

\section{Mechanism in Action of Tyrosinase}

Tyrosinase (monophenol, 3, 4-dihydroxy Lphenylalanine (L-DOPA): oxygenoxido reductase, EC 1.14.18.1) is a bifunctional copper-containing enzyme that uses molecular oxygen to catalyze the oxidation of monophenols to their corresponding odiphenols (monophenolase activity) and their subsequent oxidation to o-quinones (catecholase activity). The resulting highly reactive o-quinones auto-polymerize to form brown polyphenolic catechol melanin's. Tyrosinase is disseminated in a wide range of organisms, including bacteria, fungi, plants, insects, and other animals, and is responsible for a variety of functions: skin pigmentation in mammals, browning in plants, host defense system in arthropods, and differentiation of reproductive organs and spore formation in fungi. Many studies have been carried out to determine the enzymatic and molecular characteristics of tyrosinase from various prokaryotic and eukaryotic sources. Among these, tyrosinase from Agaricus bisporus is the most extensively investigated because this mushroom is liable to progress to enzymatic browning and pigmentation during development and post-harvest storage, which particularly decreases the commercial value of the product, and the molecular properties of tyrosinase have been provided by information from studies of $A$. bisporus tyrosinase (Konishi et al., 2007).

Tyrosinase contains a single type- 3 copper center in its active site, such as catechol oxidase from plants and hemocyanin from mollusks and arthropods. Depending on copper ion valence and linking with molecular oxygen, the copper center consists of three forms: deoxy ( $\mathrm{CuI}-\mathrm{CuI}$ ), oxy (CuII $-\mathrm{O}_{2}-\mathrm{CuII}$ ), and met activated to form a mature enzyme by proteolysis. Tyrosinase is an attractive enzyme for industrial applications: in environmental technology for the detoxification of phenol-containing waste water and contaminated soils, the production of antioxidant o-diphenols with beneficial properties as food additives and in pharmaceutical drugs, the formation of biopolymers with useful viscoelastic properties, and the improvement of food as to flavor, taste, and texture by polymerization of phenol derivatives and by cross- linking of protein-protein and protein polysaccharide. 
Tyrosinase from edible mushrooms is especially expected to have biotechnological potential in food applications because the non-toxicity of the tyrosinase is certified by historical use of mushrooms as a food. In preliminary experiments, we prepared cellfree extracts of 11 kinds of commercially available mushrooms and measured tyrosinase activity using 4-tertbutylcatechol (TBC) as a substrate (Lee et al., 1997; Konishi et al., 2007).

There is an increasing demand for different enzymes in modern industries. Besides, in spite of the outstanding progress in chemistry, there is not yet efficient reagent for synthesizing some chemical substances. Ortho-Hydroxylation of phenolic compounds is a good example of such reactions. This reaction happens easily and repeatedly in almost all organisms and results in formation of important biochemical such as neurotransmitters of Ldopa family, coumestrol, polyphenolic acids and tannins and. However, chemistry has failed to introduce a decent reagent for this seemingly simple reaction up to now. Except tyrosine hydroxylase, tyrosinase carries out this reaction effortlessly and efficiently Therefore, a great deal of effort has been put on applying this enzyme in biotransformation of the desired phenolic compounds to their corresponding orthodihydroxy substances both in aqueous and non-aqueous media.

\section{Phenol Degradation by Tyrosinase}

Tyrosinase is a cuproenzyme responsible for formation of the pigments of skin, hair, and eye. Tyrosinase is found through- out the phylogenic tree and has been extracted from different sources such as fungi, fruits, and mammalian melanoma tumors. However, edible mushroom is considered as a clean, enriched, and cheap source of this enzyme.
The active site of mushroom tyrosinase (MT) is close to the surface of the protein skeleton. Hence, it is accessible to small and large substrates. There are considerable reports indicating the great potential of this enzyme for food, medicine and agricultural industries as well as analytical and environmental purposes Furthermore, MT like mammalian tyrosinase has a tetrameric structure and can be used for clinical purposes. All these features have made MT an apt tool for today's biotechnology. For this purpose, we decided to develop simple procedure for purification of this enzyme from fresh edible mushroom (Agaricus bisporus) (Schroder et al., 2006).The enzyme tyrosinase is a member of the tyrosinase related protein family and is found throughout the phylogenetic scale of living organisms. Tyrosinase performs a variety of functions in these organisms ranging from pigmentation to defense to sclerotization. The enzyme catalyzes at least two different reactions using phenolic compounds and molecular oxygen as substrates. These reactions include hydroxylation of monophenols to form ortho-diphenols (monophenolase or cresolase activity) and oxidation of diphenols to form ortho-diquinones (diphenolase or catechol oxidase activity). It has also been suggested that tyrosinase can oxidize 5,6- dihydroxyindole to the 5,6dihydroxyquinone. There are several oxidases that can use phenolic substrates present in mushrooms, but tyrosinase is probably the principal enzyme involved in browning reactions in Agaricus bisporus. For example, tyrosinase, laccase, and peroxidase were detected in Portabella mushrooms, a brown strain of Agaricus bisporus, but tyrosinase was present in larger amounts than either laccase or peroxidase. These authors also found that more tyrosinase was present in the stalk than in the cap skin, gill tissue, or cap flesh 
tissue. However, it is difficult to accurately determine quantitative tyrosinase activities associated with different tissues because of latent and active enzyme forms, the difficulty of determining protein content, and different isoform composition at different developmental stages also showed that crude extracts of different mushroom tissues showed a slightly different isoform composition after analysis by isoelectric focusing (IEF). Crude gill tissue extracts showed less of the low pI isoforms and more of the higher $\mathrm{pI}$ isoforms. Tyrosinase isolated from cap skin and cap flesh of Portabella mushrooms and from Agaricus bisporus has a subunit molecular weight of around 43-48 kDa, although latent enzyme can be isolated with a higher molecular weight using freshly picked/frozen Agaricus bisporus tissue (Konishi et al., 2007).

Gill tissues contain abundant melanized spores in mature mushrooms. G-glutaminyl4-hydroxybenzene (GHB) is considered to be the main substrate for melanogenesis catalyzed by tyrosinase/phenolase in Agaricus bisporus. Tyrosinase has been shown to hydroxylate GHB into gglutaminyl-3,4-dihydroxybenzene (GDHB) and then to oxidize GDHB into gglutaminyl-3,4-benzoquinone during the melanization process of sporogenesis (Seo et al., 2003). However, the fate and physiological role of GHB may be different in mycelium. Tyrosinase has been located in "tyrosinase vesicles" associated with cell walls and perhaps in cell wall regions containing amorphous melanin as well. Since melanin deposition in fungi takes place in extracellular locations, tyrosinase may play a key role in the development of the fungal cell wall constituents. Perhaps pigmentation in the different extracellular cell wall locations and in spores relies on different tyrosinase isoforms or isoforms with different substrate specificities (Konishi et al., 2007).

\section{Purification and Production of Mushroom Tyrosinase}

Commercial mushroom tyrosinase was first partially purified by Anion Exchange Chromatography using diethylaminoethyl (DEAE) as the resin. Next, the enzyme was purified by Ion Exchange Chromatography using hydroxyapatite (HA) as the resin. Then, Hydrophobic Chromatography was carried out to further purify the enzyme; Phenyl Sepharose (PS) was used as the resin (Schroder et al., 2006).

Approximately half of the enzyme was latent in extracts prepared in the absence of protease inhibitors. Preparation of extracts in the presence of protease inhibitors that would inhibit serine proteases, metalloproteases, and cysteine proteases using PMSF, EDTA, and E-64, respectively, did not increase the percentage of latent enzyme appreciably although the total activity increased. Use of pepstatin to inhibit acidic proteases had no appreciable effect on increasing latent activity. These results indicate that at least half of the enzyme had already been converted into an active form in the tissue samples irrespective of what type of inhibitor was used. Presumably this variation is a result of limited proteolysis or partial unfolding of the latent enzyme according to the model of the maturation and activation of latent Agaricus bisporus mushroom tyrosinase proposed by. In contrast, were able to isolate a latent tyrosinase from freshly harvested and frozen Agaricus bisporus mushrooms without the use of protease inhibitors. This latent enzyme could be activated in vitro by trypsin, a serine protease (Fan and Flurkey, 2004).

Tyrosinase is an enzyme that hydroxylates monophenols and oxidizes diphenols. It functions in pigmentation, protection and 
causes browning reactions. This enzyme can be found in microorganisms, plants, fungi and animals. It has different functions in different organisms. The tyrosinase being studied in this research is extracted from mushroom. Commercial mushroom tyrosinase was used in the experiments, but it is not available in pure form. It is difficult to purify mushroom tyrosinase in large amounts. If tyrosinase was purified in large amount, structural studies of the enzyme could be carried out. Purified tyrosinase could also be used to construct a better model system for its inhibitors. In addition, mushroom tyrosinase can be used as a model for human tyrosinase Commercial mushroom tyrosinase was first partially purified by Anion Exchange Chromatography using Diethylaminoethyl (DEAE) as the resin. Next, the enzyme was purified by Ion Exchange Chromatography using hydroxyapatite (HA) as the resin (Schroder et al., 2006). Then, Hydrophobic Chromatography was carried out to further purify the enzyme; Phenyl Sepharose (PS) was used as the resin. However, the tyrosinase obtained after these techniques which are widely used in protein purification are salting-out by high concentrations of ammonium sulfate and ion-exchange chromatography on a column of a resin such as Diethylaminoethyl cellulose (DEAEcellulose). When a fractionation with ammonium sulfate precedes ion-exchange chromatography in a purification procedure, it is often necessary to remove excess salt before application of the protein mixture to the column. This is done to ensure that the ionic strength of the solution is sufficiently low for adsorption of at least some of the proteins in the mixture. However, Ueda et al.(2003) observed that the bacterialironproteinsrubredoxin and ferredoxin arc retained by a column of DEAE-cellulose when they are applied in a solution that contains a high concentration of ammonium sulfate. Both proteins are eluted from the column when the salt concentration is decreased. Since they are eluted by different concentrations of salt, rubredoxin and ferredoxin can be separated by this method. A number of other proteins also show this behavior on DEAE cellulose equilibrated with high concentrations of ammonium sulfate, and in some cases the phenomenon has been used for enzyme purification. This paper describes conditions under which proteins are retained by and eluted from DEAE-cellulose equilibrated with ammonium sulfate; they feel that the method may be of general use in protein purification (Mayhew and Howell, 1971).

Immobilized metal ion affinity chromatography (IMAC), also called metal chelate chromatography, has been used widely for purification of proteins since its introduction. Interactions of specific amino acid side chains, particularly those of histidine, cysteine, and tryptophan, with transition metal ions that are bound to the chelating groups of a chromatographic resin result in retention of proteins on the chromatographic column. IMAC has been used to examine the relationship between amino acid side chain surface topography of proteins and binding selectivity. One of the more recent applications of IMAC is the purification of recombinant proteins containing histidine tags (Powell et al., 2007).

Historically, the mushroom enzyme has been purified by adsorption or precipitation methods followed by ion exchange or sizeexclusion chromatography. Most purifications of the enzyme have used anionexchange chromatography followed by chromatography on hydroxyapatite. These methods can separate different isoforms of the enzyme. After Flurkey and Jen discovered that the enzyme can bind to 
Phenyl Sepharose through hydrophobic and affinity interactions, chromatography on Phenyl Sepharose has been included in many purification schemes of the enzyme. In spite of the large number of reported purifications, tyrosinase still continues to be isolated in small amounts and often contains contaminants. In particular, commercial mushroom tyrosinase preparations, which have been used in numerous substrate and inhibition studies, contain many contaminants and may require extensive purification to obtain an enzyme sample free of other proteins. IMAC could serve as an alternative purification method for removing some of the contaminating proteins in these tyrosinase preparations (Powell et al., 2007).

IMAC has already been used with some success in the purification or partial purification of PPO from artichokes, carrots, apples, and grapes. In each of these examples, $\mathrm{Cu}^{2+}$ was immobilized on a chromatographic column by chelation with iminodiacetic acid (IDA) resin. Multiple peaks of enzyme were noted depending on the elution conditions, and multiple forms of the enzyme were often present. For example, Richard-Forget et al. have an extensive description of the purification of apple PPO using hydrophobic, affinity, and metal chelate chromatography (IMAC). In their study multiple peaks of the enzyme were observed after IMAC, but no clear separation of isoforms was noted. They concluded that ion-exchange chromatography was more selective than IMAC for separating PPO isoforms. In a somewhat different approach, purified tyrosinase from the skin of the white silky fowl in a single step using $\mathrm{Cu}^{2+}$ coordinated to the Diethylaminoethyl group of DEAE cellulose. They concluded that retention of tyrosinase on the column was due to the formation of a tyrosinase $-\mathrm{Cu}^{2+}$-DEAE complex. The complex was disrupted and the enzyme was eluted under acidic conditions. In a similar approach using $\mathrm{Cu}^{2+}$ DEAE chromatography, purified a tyrosinase from Bacillus thuringiensis. To explore the possibility that IMAC could remove protein contaminants from commercial mushroom (Agaricus bisporus) tyrosinase, they immobilized a variety of metal ions $\left(\mathrm{Cu}^{2+}, \mathrm{Ni}^{2+}, \mathrm{Co}^{2+}, \mathrm{Zn}^{2+}\right)$ on IDA columns and tested their ability to separate tyrosinase from other protein contaminants. $\mathrm{Cu}^{2+}$ DEAE columns were also prepared and tested in a similar manner. Using the crystal structure of sweet potato catechol oxidase (CO) as a template, a homology model for the structure of mushroom tyrosinase was developed and used to determine which amino acid residues might be available for binding to the immobilized metal ions (Powell et al., 2007)

Hydroxyapatite is a form of calcium phosphate that has long been used in the chromatographic separation of proteins and DNA. Hydroxyapatite is best known as a crystalline material but is now available in a range of ceramic derivatives that are vastly superior in terms of flow rate, stability, and reproducibility over many cycles of use. These developments have led to a renewed interest in the use of this media with unique separation properties.

The adsorption of proteins to hydroxyapatite is complicated because it involves both anionic and cationic exchange. Proteins are most commonly adsorbed in a low concentration (10-25 mM) of phosphate buffer, although some acidic proteins are adsorbed only if loaded in water, saline, or a nonphosphate buffer. Proteins are usually eluted by an increasing phosphate gradient, although gradients of $\mathrm{Ca} 2 \mathrm{p}, \mathrm{Mg} 2 \mathrm{p}$, or $\mathrm{Cl} \grave{A}$ ions are also useful, especially for the selective elution of basic proteins. When using phosphate, acidic proteins are more 
readily eluted than basic proteins, although the phosphate concentration required to elute any protein can be reduced by raising the $\mathrm{pH}$ (Schroder et al., 2003). A mixture of proteins bound to hydroxyapatite can be fractionated by a series of phosphate wash steps of increasing pH (Konishi et al., 2007). However, phosphate is a weak buffer outside the 6.0-7.5 $\mathrm{pH}$ range, yet strong buffering is desirable when using hydroxyapatite to avoid the local $\mathrm{pH}$ changes that occur in the highly polarized environment at the surface. Analysis of the effect of altering the $\mathrm{pH}$ upon protein elution would therefore benefit from improved buffering because the buffering capacity of the phosphate rapidly decreases away from neutrality. In the present paper, a simple procedure is described that extends the phosphate-mediated hydroxyapatite elution of proteins over a wider $\mathrm{pH}$ range while maintaining a properly buffered environment. Others have not previously reported the approach of using a second buffer system to broaden the $\mathrm{pH}$ range of separation with hydroxyapatite media (Schroder et al., 2003).

Affinity chromatographythe term which has been coined to describe a separation on a special solid phase containing a ligand which binds the molecule to be purified. The process usually applied to biological materials and to interactions such as those between antigens and antibodies, nucleic acids and nucleotides, and proteins and protein-binding substances. As applied to enzyme purification, the method utilizes the specific binding between an enzyme and an inhibitor, a cofactor, or a substrate analog as a means of selectively removing an enzyme from a complex protein mixture (Powell et al., 2007).

Suitable polymeric materials are chemically modified to introduce pendant groups with enzyme-binding properties; often an intermediary molecule required between the polymeric backbones and the ligand group to avoid steric hindrance to enzyme-ligand interaction. The modified polymer then can be used under suitable conditions in the form of a packed column for enzyme retention. Enzyme recovery, and thus column regeneration, is brought about by washing with an appropriate solution (with, e.g., altered $\mathrm{pH}$, altered salt concentration, or added substrate) so that the enzyme is eluted. There are several practical guidelines for the support phase: It should contain functional groups which can react readily with a wide range of Uganda, It should exhibit good flow properties (low compaction) in a packed bed, It should have very few ionic or other group which can adsorb protein nonspecifically, and It should provide free access to the bulky enzyme molecules by containing large pores and by having a high fractional porosity. This enzyme's kinetics and function, they felt that an AC column which was effective with tyrosinase might well be suitable for other phenol oxidizing enzymes, which we are also in the process of isolating (Sean et al., 1973).

\section{Application of Tyrosinase in Environment Industry}

Kameda et al., in (2014) assessed that Phenols are toxic pollutants found in industrial wastes imposing several risks to human health. Tyrosinase is oxygen as deoxyreductase found in several life forms, like the mushroom Agaricus bisporus. This enzyme is readily available from this fungal tissue leading to high activity extracts without extensive purification, thus suggesting its potential as a biocatalyst for applications involving bio modification of phenols or bioremediation of phenolpolluted waters. The in nature fungi was also able to remove $90 \%$ of phenol, 
demonstrating its viability as a biocatalyst in bioremediation process.

Chen and Flurkey (2002) also supported the above results through the results of their experiments that Phenols are toxic pollutants found in industrial wastes imposing several risks to human health. Tyrosinase (EC 1.14.18.1) is an oxygenaseoxy reductase found in several life forms, like the mushroom Agaricus bisporus. This enzyme is readily available from this fungal tissue leading to high activity extracts without extensive purification, thus suggesting its potential as a biocatalyst for applications involving biomodification of phenols or bioremediation of phenol-polluted waters (Xu and Yang, 2013). The purpose of this work was to employ a crude extract from the Agaricus bisporus mushroom and its biomass for the removal of phenol from polluted water. Experiments were carried out without $\mathrm{pH}$ control. Chatoyant was used as a coagulant, but no significant improvement was observed. The in nature fungi was also able to remove $90 \%$ of phenol, demonstrating its viability as a biocatalyst in bioremediation process.

Flurkey in (1991) demonstrated that Isoenzymes of tyrosinase were identified in mushrooms using vertical polyacrylamide gel isoelectric focusing in conjunction with a $\mathrm{pH}$ 4-7gradient. In the MB strain, the epidermis, cap flesh, gill, and stalk tissue showed similar isoenzyme after isoelectric focusing, but the distribution of staining intensity appeared different for each type tissue (Espin et al., 1999). Younger developmental stages of $\mathrm{MB}$ mushrooms showed a different isoenzyme profile than older ones. Mature mushrooms cut at the stripe showed a different distribution of isoenzyme staining than those not cut at the stripe, indicating possible activation of latent enzyme or new synthesis of specific tyrosinase isoenzymes
Flurkey and Jan, (1980) proved that Mushroom tyrosinase was subjected to affinity and hydrophobic chromatography on 2-mercapto benzimidazole, 2-mercapto benzothiazole, methimazole, mimosine, and tropolone ligands coupled to solid supports. Mercapto benzimidazole, mercapto benzothiazole, and methimazole columns coupled to $\mathrm{CNBr}$ activated Sepharose and mimosine coupled to epoxy activated Sepharose were capable of retaining significant amounts of the enzyme under affinity chromatography conditions. Only mercapto benzimidazole and mercapto benzothiazole columns retained significant amounts of the enzyme under hydrophobic chromatography conditions. The enzyme was also adsorbed on to phenyl and phenylbutylamine columns under hydrophobic conditions (Schroder et al., 2003). Increasing amounts of the enzyme were bound to several alkyl columns under hydrophobic condition sand the binding increased as the initial salt concentration increased. Mushroom tyrosinase was also adsorbed onto two antibody columns. One column retained the holoenzyme while the other column retained smaller molecular weight proteins related to the enzyme. All resins which bound the enzyme resulted in significant purification and removal of contaminating proteins compared to crude samples. These results suggest that mushroom tyrosinase can be purified and characterize during inhibitors, phenolics, alkyl groups, and antibodies coupled to solid supports.

Khan et al.,(2005) verified that Use of immobilized and stable enzymes has immense potential in the enzyme analysis of clinical, industrial and environmental samples. But their wide spread use is limited due to the high cost of their production. In the present study, an effort has been made to immobilize tyrosinase directly from 
ammonium sulphate precipitated proteins of the mushroom (Agaricus bisporus) on a polyclonal antibody bound to Seralose 4B support.

Amuneke et al. (2011) confirmed that Pleurotus ostreatus an edible mushroom was cultivated from agricultural wastes such as sawdust, cassava peel, cotton waste, dry plantain, palm oil chaff, and vegetable. Standard microbiological and chemical methods were adopted to determine the microbial and proximate quality of the substrates. The cultivation of this species involved isolation of the mushroom, preparation of the spawn and substrate, spawning, incubating, cropping, harvesting and packaging. The study showed that sterilization and watering of the mushroom bag was a limiting factor in the growth of the mushroom. Organisms such as Bacillus spp, Mucor spp and Rhizopus spp were implicated during substrate preparation and fruiting of the mushroom. All the substrates supported the growth of fungi except vegetable. Fan and Flurkey in 2003 showed that a group of tyrosinase isoforms with isoelectric points between 4.9 and 5.2 was isolated from gill tissue of Portabella mushrooms. Use of protease inhibitors was not able to increase the amount of latent forms significantly in crude extracts or to preserve latent tyrosinase activity during purification. The tyrosinase in gill tissue extracts showed latent activity above $\mathrm{pH} 5.5$ and suppressed or displayed no latent activity below $\mathrm{pH} 5.5$ when assayed in the presence of SDS. The purified isoforms showed monophenolase activity toward 4hydroxyanisole but practically no activity toward tyrosine or tyramine (Zhang and Flurkey, 1999). The purified isoforms showed greater activity toward catechol than 4-methylcatechol, DOPA, DOPAmine, chlorogenic acid, t-butylcatechol, or catechin. Papa et al., in 1994 reported that method for tyrosinase (EC1.14.18.1) extraction, purification and separation from Agaricus bisporus proposed. They also reported that Modifications primarily concern: (a) enzyme recovery by performing pellet re-extractions, (b) preparative isoelectric focusing purification replacing a gel-permeation, (c) use of polyvinylpyrrolidone to remove contaminant "brown proteins", instead of basic $\mathrm{Pb}$ acetate. With this procedure, it is possible to enhance the amount of tyrosinase recovered, thus reducing the quantity of mushrooms required $(90 \%$ less). The enzyme obtained displays a better degree of purification (between 81- and 92-fold), and the total extraction time is lower than that of the original technique.

Ikehata and Nicell (2000) presented the data showing that Mushroom tyrosinase is an alternative to peroxidase enzymes for the catalytic removal of phenolic compounds from wastewaters. Güray and Mohamed (2013) displayed the result showing that Tyrosinase is a key enzyme in the synthesis of melanin and is widely distributed in animals, plants, and microorganisms. As excessive melanin production causes not only hyper pigmenting effects on human skin but also melanosis in various foods, an inhibitor of tyrosinase has become of interest lately from a practical point of view. Suzuki et al. (1992) experimentally proved that Thermostable p-tyrosinase was extracted from an obligate symbiotic and thermophilic bacterium, Symbiobacterium thermophilum, which grows only in cocultivation with a specific thermophilic Bacillus sp., strain $\mathrm{S}$.

Tyrosinase catalyses the o-hydroxylation of monophenols into their corresponding odiphenols (monophenolase or tyrosine hydroxylase (TH) activity), and the oxidation of $\mathrm{O}$ - diphenols to o-quinones 
(diphenolase or DOPA oxidase activity), using molecular oxygen, which then polymerize to form brown or black pigments. The synthesis of o-diphenols (catechols) is a potentially valuable catalytic ability and thus tyrosinase has attracted a lot of attention with respect to biotechnological applications. The mechanism of action and the catalytic cycles of the TH and L-DOPA oxidase activities of the enzyme have been studied with respect to the activity towards 1-tyrosine and L-DOPA. The enzyme contains a dinuclear type-3 copper centre, in which two copper ions are closely spaced and coordinated each by three histidines through the nitrogen atoms. Cloning, sequencing and expression studies have been carried out on tyrosinase from a number of bacterial sources including Streptomyces antibiotics, Streptomyces lavendulae, Rhizobiumetli and Pseudomonas maltophila generating information about the enzyme structure and function. The enzyme has also been purified from many sources such as mouse melanoma, mushroom, plants, animals and bacteria.

Martin et al. (2008) conducted the experiments showing that the conversion of phenols to o-diphenols by tyrosinase is a potentially attractive catalytic ability and thus tyrosinase has attracted a lot of attention with respect to its biotechnological application as the catechol products are useful as drugs or drug pythons, e.g. LDOPA. In order to assess the catalytic potential of tyrosinase we have taken different sources of the enzyme and directly compared their catalytic efficiency (kcat/Km) towards substituted phenols. The determination of the kinetic constants is the first step in the assessment of tyrosinase as a biocatalyst. We have focused on fluorinated phenols as fluorinated compounds possess interesting biological activity (e.g. enzyme inhibition, affect energy generation processes and cell to cell communication) and the vast majority of the research into the transformation of halogenated substrates has focused on chlorinated and brominated substrates.

Seetharam and Saville (2003) stated that degradation of phenol by tyrosinase immobilized on chemically modified sodium alumina silicate $(\mathrm{NaA})$, calcium alumina silicate $(\mathrm{CaA})$, and silica gel was studied. Phenol conversion by immobilized tyrosinase ranged between B15\% and 60\%, depending upon the initial phenol concentration, $\mathrm{pH}$, and enzyme loading. Ikehata and Nicell in 2000 verified that Pleurotus is a genus of gilled mushroom, commonly called as oyster mushroom. They belong to kingdom Fungi, family Tricholomataceae and class Basidiomycetes. They are found in tropical/temperate climates at a temperature range of $22-30^{\circ} \mathrm{C}$ and humidity $80-95 \%$. Oyster mushrooms are the second most common mushroom produced in India, as well as worldwide. These mushrooms are of edible as well as medicinal importance.

Ingebrigtsen et al. (1988) reported that Tyrosinase is a copper-containing enzyme that has been found widely distributed in microorganisms, plants and animals. Tyrosinase Browning Reactions in fruits and vegetables are recognized as a serious problem in the food industry. In many fruit and vegetable products, these reactions are associated with peroxidase and polyphenoloxidase. In mushrooms, the major enzyme responsible for the brown infraction is tyrosinase although many mushroom species also contain lactase activity. The control of this enzyme in mushrooms is desirable in light of the recent ban on the use of sulfites to delay browning reactions. The enzyme has also been studied during aging and during post-harvest 
treatment sand or storage. Investigations have also been carried out to examine ways to increase the shelf life and quality of mushrooms. Surprisingly, much less information is available on the activity, isoenzymes and location of tyrosinase in developing mushrooms. Recently, reported that the skin contained more enzyme than the flesh based on either a fresh weight or on a protein basis.

Kumar and Flurkey (1991) perceived that several lots of commercial tyrosinase preparations have been examined with regard to their enzyme activity, isoenzyme composition and purity. Enzyme activity toward catechol, L-DOPA and tyrosine showed significant variations from lot to lot and activation by SDS. Distribution of isoenzyme forms also varied from lotto lot. Comparisons of electrophoretic and isoelectric focusing protein profiles showed considerable differences and distributions of the proteins in each sample. Tyrosinase appeared to be a minor component in each preparation when compared to a partially purified enzyme (Zhang and Flurkey, 1999). Investigators using commercial tyrosinase should exercise caution in interpreting data due to the presence of different isoenzyme forms, their distribution in various lots, and the presence of numerous other proteins. They professed that The P-isozyme of mushroom tyrosinase is composed of an equilibrium mixture of polymeric forms with the tetramer predominating.

Ensuncho et al.,(2005) conducted the experiments to investigate the removal of aqueous phenol using immobilized enzymes in both bench scale and pilot scale threephase fluidized bed reactors. The enzyme used in this application was a fungal tyrosinase immobilized in a system of chatoyant and alginate. The immobilization matrix consisted of a chatoyant matrix cross- linked with glutaraldehyde with an aliginatefilledpore space. This support matrix showed superior mechanical properties along with retaining the unique adsorptive characteristics of the chatoyant. Adsorption of the o-quinone product by the chatoyant reduced tyrosinase inactivation that is normally observed for this enzyme under these conditions. Some of these preparations were homogeneous, or almost homogeneous, by either electrophoresis or ultracentrifugation; one was homogeneous by both criteria. Haghbeen et al. (2004) presented that a simple preparative method was developed for purification of Tyrosinase from edible mushroom (Agaricus bisporus). Since civilization will most probably continue to be accompanied by the production of hazardous waste materials, it is necessary to develop efficient strategies for waste management. Biotechnology for hazardous waste management involves the development of biological systems that catalyze the detoxification, degradation or decontamination of environmental pollutants. In future technologies, microbial systems might be the potential tools to deal with the environmental pollutants.

\section{Conclusion}

Phenolic compounds are perilous pollutants that are noxious at relatively low concentration and cause pollution; it must be impassive from the environment. The custom of microbial catalysts in the biodegradation of phenol has trailblazingimplication during the bygone three decades. It has been initiated that large numbers of microbial enzyme exist in virtually all natural environments, which boost particular reaction which can be castoff for commercial purpose. Phenol being such menacingbiodegradable materials can be degraded at faster rate by extra cellular enzymes. Mushroom tyrosinase has been 
expansively studied in crude extracts, purified forms and in commercial preparations with esteem to its physical and enzymatic properties for application in environment industry. Mushroom tyrosinase can be used for the catalytic elimination of phenolic compounds from environment.

\section{Acknowledgement}

Authors are thankful to Head, Dept. of P.G. Studies and Research in Biological Science, R.D. University, Jabalpur for providing Laboratory facilities.

\section{References}

Amuneke, E.H., Dike, K.S., Ogbulie, J.N. 2011. Cultivation of Pleurotus ostreatus: An edible mushroom from agro base waste products. J. Microbiol. Biotechnol. Res., 1: 1-14.

Chen, L., Flurkey, W.H. 2002. Effect of protease inhibitors on the extraction of Crimini mushroom tyrosinase isoforms. Phytochem., 5: 109-120.

Ensuncho, L., Cuenca, M.A., Legge, R.L. 2005. Removal of aqueous phenol using immobilized enzymes in a bench scale and pilot scale three-phase fluidized bed reactor. Bioprocess and Biosystems Engineering, 27: 185-191.

Espín, J.C., Leeuwen, J.V., Wichers, H.J. 1999. Kinetic study of the activation process of a latent mushroom (Agaricus bisporus) tyrosinase by serine proteases. J. Agri. Food Chem., 47: 3509-3517.

Fan, Y., Flurkey, W.H. 2004. Purification and characterization of tyrosinase from gill tissue Portabella mushrooms. Phytochem., 65: 671-678.

Flurkey, W.H., Jen, J.J. 1980. Hydrophobic adsorption chromatography of peach polyphenol oxidase. J. Food Sci., 5: 1622-1624.
Güray, M.Z., Mohamed, S. 2013. A new thermophilic polyphenol oxidase from bacillus sp.: partial purification and biochemical characterization. $J$. Proteins and Proteomics, 4: 11-20.

Hagbeen, K., Azii, F.R., Karkhane, A.A., Borojerdi, S.S. 2004. Purification of tyrosinase from edible mushroom. Iranian J. Biotechnol., 2: 3.

Ikehata, K., Nicell, J.A. 2000. Characterization of tyrosinase for the treatment of aqueous phenols. Biores. Technol., 74: 191-199.

Ingebrigtsen, J., Flurkey, W.H. 1988. Affinity and Hydrophobic chromatography of mushroom tyrosinase. Phytochem., 7: 1593-1599.

Khan, A.F., Akhtar, S., Husain, Q. 2005. Simultaneous purification and immobilization of Mushroom Tyrosinase on an Immouno affinity support Process. Biochem., 40: 23782386.

Konishi, Y.K., Tsuji, Y.M., Hatana, S., Asanuma, M., Kakuta, D., Kawano, T., Mukouyama, E.B., Goto, H., Suzuki, H. 2007. Purification, Characterization, and Molecular Cloning of Tyrosinase from Pholiotanameko. Biosci. Biotechnol. Biochem., 71: 1752-1760.

Koshy, J., Chandran, N., Nambisan, P. 2012. Biodegradation of phenol using spent substrate of Pleurotus sp. J. Pharma. Sci., 1: 656-661.

Kulkarni, S.J., Kaware, J.P. 2013. Review on Research for removal of phenol from wastewater. Int. J. Sci. Res., 3: 2750-3153.

Kumar, M., Flurkey, W.H. 1991. Activity, isoenzymes, and purity of mushroom tyrosinase in commercial preparations. Phytochem., 30: 3899-3902.

Lee, J.L., Kong, K.H., Cho, S.H. 1997. Purification and Characterization of Tyrosinase from SolanumMelongena. J. Biochem. Mol. Biol., 30: 150-156. 
Martin, L.B., Nikodinovic, J., Mahen, A.M., Vijgenboom, E., O'connor, K.E. 2008. Assessing the catalytic activity of three of the oxidation of mono and difluornated monophenols. Enzyme and Microbial Technol., 43: 297-301.

Mayhew, S., Howell, L. 1971. Chromatography of protein on DEAE Cellulose. Anal. Chem., 41: 466-470.

Papa, G., Pessione, E., Leone, V., Giunta, C. 1994. Agaricus Bisporus Tyrosinase I- progress made in preparative methods. Int. J. Biochem., 26: 215-221.

Paul, S., Chakraborty, S., Ali, N., Ray, D.P. 2015. Arsenic distribution in environment and its bioremediation: A review. Int. J. Agri. Environ. Biotechnol., 8: 189-204.

Powell, A., Siu, N., Inlow, J.K., Lfurkey, W.H. 2007. Immobilized Metal Affinity Chromatography (IMAC) of Mushroom Tyrosinase. Physical Properties. J. Chem., 1: 1.

Schroder, E., Jonsson, T., Poole, L. 2003. Hydroxyapatite chromatography: altering the phosphate-dependent elution profile of protein as a function of pH. Anal. Biochem., 313: 176-178.

Schroder, E., Jonsson, T., Poole, L. 2003. Hydroxyapatite chromatography: altering the phosphate-dependent elution profile of protein as a function of pH. Analytical Biochem., 313: 176178.

Sean, P., O"Eill, J.D., Graves, J., James, Ferguson, J. 1973. Affinity
Chromatography of Mushroom Tyrosinase. J. Macromol. Sci. Part AChem:Pure and Appl. Chem., 1159: 1166.

Seetharam, G.B., Saville, B.A. 2003. Degradation of phenol using tyrosinase immobilized on siliceous support. Water Res., 37: 436-440.

Seo, S.Y., Sharma, V.K., Sharma, N. 2003. Mushroom tyrosinase: recent prospects. J. Agri. Food Chem., 51: 2837-2853.

Suzuki, S., Thirahara, J.K., Shim, S., Horinouchi, Beppu, T. 1992. Purification and Properties of thermostable B-Tyrosinase from an obligately symbiotic thermophile, symbiobacterium thermophilum.

Biosci. Biotechnol. Biochem., 56: 8489.

Ueda, E.K.M., Gout, P.W., Morganti, L. 2003. Current and prospective applications of metal ion-protein binding. J. Chromatography A, 988: 123.

Xu, D.Y., Yang, Z. 2013. Cross-Linked Tyrosinase aggregates for elimination of phenolic compounds from wastewater. Chemosphere, 92: 391398.

Zhang, X., Flurkey, W.H. 1999. Purification and partial characterization of tyrosinase isoforms from cap flesh of Portabella mushrooms. J. Food Biochem., 23: 95-108.

\section{How to cite this article:}

Anjana Sharma and Juhi Sharma. 2016. A Review on Amelioration of Mushroom Tyrosinase for Phenol Degradation to Combat Environment Pollution. Int.J.Curr.Res.Aca.Rev.4(4): 29-43. doi: http://dx.doi.org/10.20546/ijcrar.2016.404.004 